\title{
Adaptive Fault-Tolerant Control of a Hybrid VTOL UAV against Actuator Faults and Model Uncertainties under Fixed-Wing Mode
}

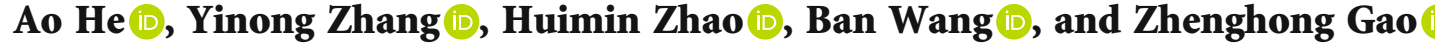 \\ School of Aeronautics, Northwestern Polytechnical University, Xi'an 710072, China \\ Correspondence should be addressed to Ban Wang; wangban@nwpu.edu.cn
}

Received 9 October 2021; Accepted 24 December 2021; Published 10 January 2022

Academic Editor: Youmin Zhang

Copyright () 2022 Ao He et al. This is an open access article distributed under the Creative Commons Attribution License, which permits unrestricted use, distribution, and reproduction in any medium, provided the original work is properly cited.

\begin{abstract}
This paper proposes an adaptive fault-tolerant control strategy for a hybrid vertical take-off and landing (VTOL) unmanned aerial vehicle (UAV) to simultaneously compensate actuator faults and model uncertainties. With the proposed adaptive control schemes, both actuator faults and model uncertainties can be accommodated without the knowledge of fault information and uncertainty bounds. The proposed control scheme is constructed with two separate control modules. The low-level control allocation module is used to distribute the virtual control signals among the available redundant actuators. The high-level control module is constructed with an adaptive sliding mode controller, which is employed to maintain the overall system tracking performance in both faulty and uncertain conditions. In the case of actuator faults and model uncertainties, the adaptive scheme will be triggered to generate more virtual control signals to compensate the virtual control error and maintain the desired system tracking performance. The effectiveness of the proposed control strategy is validated through comparative simulation tests under different faulty and uncertain scenarios.
\end{abstract}

\section{Introduction}

In recent years, with the development of unmanned aerial vehicle (UAV) technology, more and more UAVs have been developed and employed for various practical applications, such as payload transportation [1], aerial surveillance [2], and border monitoring [3]. For many of the industrial applications, vertical take-off and landing (VTOL) is a basic requirement for UAVs. In addition, in order to accomplish the assigned mission more efficiently, UAVs are expected to fly with long endurance. In this case, different types of hybrid VTOL UAVs have been developed, such as tilt-wing UAV, tilt-rotor UAV, tailsitter UAV, and canard rotor/wing (CRW) UAV, which combines the advantages of both rotary-wing and fixedwing UAVs to achieve a wider flight envelope [4]. The extensive applications of VTOL UAVs in various fields have attracted many researchers to work on such type of UAVs. The higher hovering efficiency, cruising speed, and the payload capacity are the major concerns of the research works in the literature. In [5], the investigation of analytical behavior of fixed-wing VTOL aircraft with gas-driven fan propulsion mechanism is performed, and the proposed propulsion system can provide a range of load for low-speed operations and the energy loss coefficient of propulsion system must be less than 0.2 for an optimized functioning of the system. In [6], a conceptual design of a transitional VTOL UAV is performed with some incomparable maneuver and attitude capabilities. In [7], a commercial hybrid VTOL UAV system with detachable and changeable wings is designed, and the related aerodynamic and structural configuration analysis is performed as well. In [8], a tail-sitter VTOL UAV is developed and tested with experimental verifications, which does not require conventional control surfaces in hovering flight mode compared to other types of tail-sitter VTOL UAVs. In [9], a transition flight modeling of a tilt-rotor VTOL UAV is presented which specially considers the aerodynamic effect of propeller-induced airstream. Based on the developed model, a transition state scheduler is designed and verified through simulation results. In [10], a systematic design approach for longitudinal full envelope velocity control of a small tilt-wing hybrid UAV is proposed which includes dynamic modeling, system 
identification, and nonlinear model predictive control. In [11], the flight dynamic model of a CRW aircraft is investigated and validated through flight test data, which lays the foundation for control system design.

In terms of flight control, many control strategies have been proposed for hybrid VTOL UAVs under different flight mode, i.e., helicopter mode, airplane mode, and transition mode. In [12], a nonlinear robust controller is presented for flight mode transition control of a tail-sitter VTOL UAV based on $H_{\infty}$ control and nonlinear disturbance observer to deal with the model uncertainties. In [13], an altitude controller is designed based on active disturbance rejection control to achieve fast flight mode transition for a tilt-rotor UAV during landing phase. In [14], a two-layer cascaded control allocation approach is proposed for a VTOL UAV based on incremental nonlinear dynamic inversion method. In general, model uncertainties inevitably exist, thus, the designed flight controller is required to be robust against such uncertainties. Sliding mode control (SMC) is a robust control approach that handles uncertainties with discontinuous control strategy, which demonstrates invariance to matched uncertainties when the sliding variable stays on a predefined sliding surface. In [15], a sliding mode controller is presented for a miniature unmanned helicopter to compensate unmodeled flapping dynamics and external disturbances. In [16], a sliding mode controller is proposed for a tilt-wing UAV to stabilize its attitude and altitude.

In addition, considering the fact that in-flight faults exposed on UAVs may seriously endanger the surrounding human life and property in addition to the loss of UAV itself. With the increase of complexity of UAV systems, critical safety issues need to be specially considered. This calls for the appearance of fault-tolerant control (FTC) systems. Partial loss of control effectiveness of an actuator is a common fault that occurs in UAV systems [17], that is therefore considered in this paper. The concept of FTC has been widely employed in safety-critical systems, such as spacecraft $[18,19]$ and aircraft $[20,21]$. In [22], an adaptive faulttolerant flight controller is presented for a VTOL tail-sitter UAV and validated through experiment tests. In [23], a FTC scheme is proposed for a tilt-rotor UAV developed by Korea aerospace research institute to compensate the adverse effect of actuator faults. In [24], an integrated fault estimation and fault-tolerant control strategy is developed for a system with time-varying delays and additive faults based on a dynamic event-triggered communication mechanism. In [25], a distributed finite-time fault-tolerant containment control scheme is proposed for multiple UAVs against actuator faults. In [26], a composite adaptive disturbance observer-based decentralized fractional-order fault-tolerant control strategy is developed for UAVs against actuator faults and wind disturbances. In [27], a closed-loop control allocation scheme based on nonlinear dynamic inversion is presented to accommodate actuator faults for longitudinal control of a CRW aircraft in fixedwing mode.
Although extensive research works have been carried out for safe operation of VTOL UAVs, there are still some problems that need to be further solved. Based on the above analysis, an adaptive fault-tolerant control strategy is proposed in this paper to simultaneously compensate both actuator faults and model uncertainties for a hybrid CRW UAV. The main contributions of this paper can be briefly summarized as follows:

(1) Unlike the studies in [12-14], the proposed control strategy can simultaneously compensate the adverse effect caused by actuator faults and model uncertainties without any prior knowledge of faults and uncertainties

(2) Compared to the existing sliding mode-based faulttolerant control scheme, the proposed adaptive sliding mode control scheme can avoid overestimation of the control parameters, which contributes to alleviating the unexpected control chattering

(3) With the proposed adaptive fault-tolerant control scheme, the control parameters can be updated in real-time to compensate virtual control errors and redistribute the control signals to the available redundant actuators. The stability of the closedloop system is guaranteed theoretically

The remainder of this paper is organized as follows. Section 2 introduces dynamic modeling of the studied hybrid CRW UAV. System formulations and detailed design procedures of the proposed adaptive FTC strategy are described in Section 3. The comparative simulation results are followed in Section 4 to validate the effectiveness of the proposed control strategy. Finally, general conclusions of this paper are summarized in Section 5.

\section{Dynamic Modeling of a Hybrid CRW UAV}

The studied hybrid CRW UAV is illustrated in Figure 1. The lift of the UAV is provided by the middle main rotor, the front canard, and the rear horizontal tail together. Under airplane mode, the middle main rotor is locked as a fixed-wing aircraft. In this case, the UAV will operate like a three-surface aircraft. The forward thrust comes from the electric motor that is mounted on the front of the UAV. The yaw motion of the CRW UAV is controlled by the rudder of the vertical tail. The roll and pitch motion of the CRW UAV are controlled by moving the control surfaces of both canard and horizontal tail in opposite and same direction, respectively. Since the attitude control system of the CRW UAV is directly related to the actuators, it is considered in this paper for investigating the FTC strategy.

In order to model the CRW UAV, a body-fixed reference frame is employed and denoted as $\left(o_{b}, x_{b}, y_{b}, z_{b}\right)$. The origin of the body-fixed frame coincides with the center of gravity of the CRW UAV. By employing the Newton-Euler formulation, the dynamic equations of the CRW UAV can be expressed as follows: 

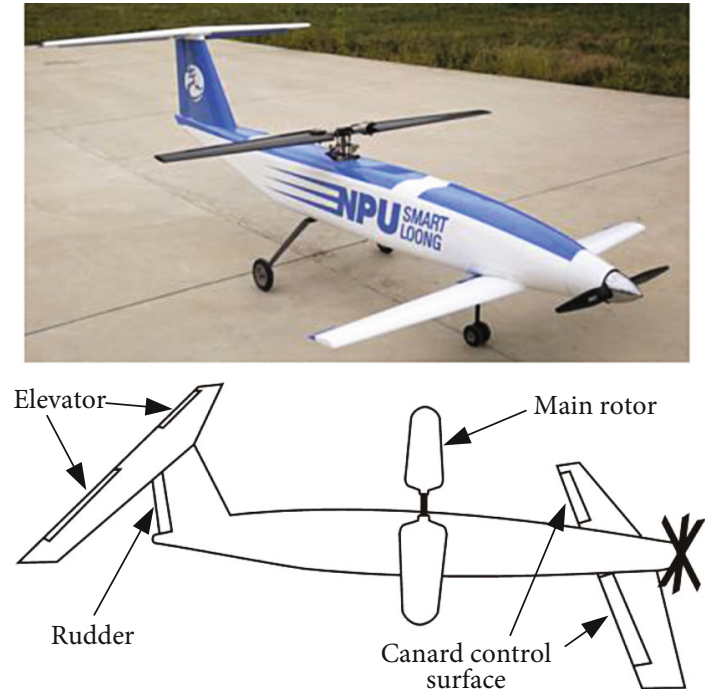

Figure 1: Illustration of the studied hybrid CRW UAV.

$$
\left\{\begin{array}{l}
\ddot{\phi}=\frac{M_{x}+\left(I_{y y}-I_{z z}\right) \dot{\theta} \dot{\psi}+I_{x z}(\dot{\phi} \dot{\theta}+\ddot{\psi})}{I_{x x}}, \\
\ddot{\theta}=\frac{M_{y}+\left(I_{z z}-I_{x x}\right) \dot{\phi} \dot{\psi}-I_{x z}\left(\dot{\phi}^{2}-\dot{\psi}^{2}\right)}{I_{y y}}, \\
\ddot{\psi}=\frac{M_{z}+\left(I_{x x}-I_{y y}\right) \dot{\phi} \dot{\theta}+I_{x z}(\ddot{\phi}-\dot{\theta} \dot{\psi})}{I_{z z}},
\end{array}\right.
$$

where $\phi, \theta, \psi$ are the roll, pitch, and yaw angle of the UAV, $I_{x x}, I_{y y}, I_{z z}$ are the moments of inertia of the UAV, and $I_{x z}$ is the product of inertia. $M_{x}, M_{y}, M_{z}$ are the resultant torques acting on the UAV along $x_{b}, y_{b}$, and $z_{b}$ axis, respectively.

Since the absolute value of $I_{x z}$ is much smaller than that of $I_{x x}, I_{y y}$, and $I_{z z}$, in order to facilitate the controller design, the components related with $I_{x z}$ in Equation (1) can be treated as disturbances. In addition, considering the fact that in practice the torques acting on the UAV may not be obtained accurately, the resultant torques are formulated as

$$
\left\{\begin{array}{l}
M_{x}=M_{x p l a n e}+M_{x c}+M_{x u} \\
M_{y}=M_{y \text { plane }}+M_{y c}+M_{y u} \\
M_{z}=M_{z \text { plane }}+M_{z c}+M_{z u}
\end{array}\right.
$$

where $M_{x p l a n e}, M_{y \text { plane }}$, and $M_{z \text { plane }}$ denote the known portions of the resultant torques; $M_{x c}, M_{y c}$, and $M_{z c}$ represent the torques generated by the deflection of control surfaces; and $M_{x u}, M_{y u}$, and $M_{z u}$ are denoted as the unknown uncertain portions of the resultant torques.
In this sense, the control-oriented model can be obtained as

$$
\left\{\begin{array}{l}
\ddot{\phi}=\frac{M_{x \text { plane }}}{I_{x x}}+\frac{\left(I_{y y}-I_{z z}\right) \dot{\theta} \dot{\psi}}{I_{x x}}+\frac{L_{\delta_{a f}} \delta_{a f}+L_{\delta_{a r}} \delta_{a r}}{I_{x x}}+\frac{M_{x u}}{I_{x x}}, \\
\ddot{\theta}=\frac{M_{y \text { plane }}}{I_{y y}}+\frac{\left(I_{z z}-I_{x x}\right) \dot{\phi} \dot{\psi}}{I_{y y}}+\frac{L_{\delta_{e f}} \delta_{e f}+L_{\delta_{e r}} \delta_{e r}}{I_{y y}}+\frac{M_{y u}}{I_{y y}} \\
\ddot{\psi}=\frac{M_{z \text { plane }}}{I_{z z}}+\frac{\left(I_{x x}-I_{y y}\right) \dot{\phi} \dot{\theta}}{I_{z z}}+\frac{L_{\delta_{r}} \delta_{r}}{I_{z z}}+\frac{M_{z u}}{I_{z z}},
\end{array}\right.
$$

where $\delta_{a f}$ is the aileron deflection of the canard control surface, $\delta_{a r}$ is the aileron deflection of the horizontal tail control surface, $\delta_{e f}$ is the elevator deflection of the canard control surface, $\delta_{e r}$ is the elevator deflection of the horizontal tail control surface, $\delta_{r}$ is the rudder deflection of the vertical tail control surface, and $L_{\delta_{a f}}, L_{\delta_{a r}}, L_{\delta_{e f}}, L_{\delta_{e r}}, L_{\delta_{r}}$ are the corresponding coefficients related to the generated torques.

\section{Adaptive Fault-Tolerant Control Strategy}

In this section, an adaptive sliding mode control strategy is designed to accommodate both actuator faults and model uncertainties for the studied hybrid CRW UAV. The schematic of the proposed control strategy is illustrated in Figure 2. There are two problems to be addressed in the following sections. The first problem is to construct an integral sliding mode controller and a control allocation strategy to guarantee system tracking performance in fault-free conditions. The second one is the problem of exploring the online adaptive schemes for compensating virtual control errors while maintaining the desired system tracking performance in the presence of actuator faults and model uncertainties.

3.1. Problem Formulation. Consider an integral chain nonlinear affine system with model uncertainties and disturbances subject to actuator faults:

$$
\left\{\begin{array}{l}
\dot{x}_{1}(t)=x_{2}(t), \\
\dot{x}_{2}(t)=F\left(x_{1}(t), x_{2}(t)\right)+H B_{u} L_{f}(t) u(t)+d(t),
\end{array}\right.
$$

where $x(t)=\left[x_{1}(t), x_{2}(t)\right]^{T} \in \mathbb{R}^{n}$ is the state vector, $B_{u} \in$ $\mathbb{R}^{p \times m}$ is the control effectiveness matrix, $u(t) \in \mathbb{R}^{m}$ is the control input vector, and $H \in \mathbb{R}^{p \times p}$ is a diagonal matrix. $d($ $t) \in \mathbb{R}^{p}$ denotes the disturbances that are assumed to be unknown and bounded as $\|d(t)\| \leq D$. The vector $F\left(x_{1}(t)\right.$, $\left.x_{2}(t)\right) \in \mathbb{R}^{p}$ is a nonlinear function that contains model uncertainties. $\quad L_{f}(t)=\operatorname{diag}\left(\left[l_{f 1}(t), l_{f 2}(t), \cdots, l_{f m}(t)\right]\right)$ is a diagonal matrix representing the level of control effectiveness of the actuators, where $l_{f j}(t)(j=1,2, \cdots, m)$ is a scalar satisfying $0 \leq l_{f j}(t) \leq 1$. If $l_{f j}(t)=1$, the $j$ th actuator works perfectly, otherwise, the $j$ th actuator suffers a certain level of fault. 


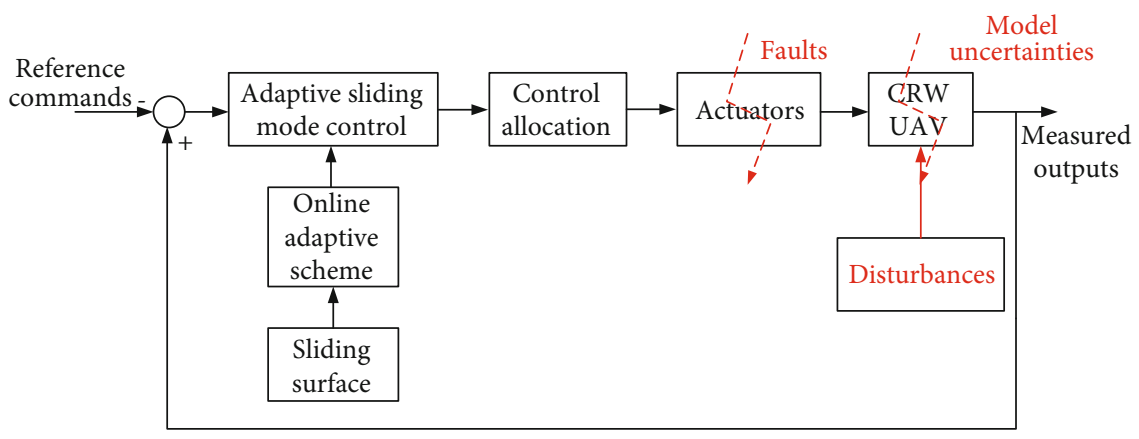

FIgURE 2: Schematic of the proposed adaptive fault-tolerant control strategy.

In order to facilitate the controller design, by defining the system states as $x=[\phi, \dot{\phi}, \theta, \dot{\theta}, \psi, \dot{\psi}]^{T}$, the nonlinear system in Equation (4) can be represented as

$$
\left\{\begin{array}{l}
\dot{x}_{2 i-1}=x_{2 i}, \\
\dot{x}_{2 i}=f_{i}(x)+h_{i} v_{i}+d_{i}, \\
v_{i}=B_{u} L_{f} u,
\end{array}\right.
$$

where $i=1,2,3$ represents each subsystem, $x_{2 i-1} \in[\phi, \theta, \psi]^{T}$, and $x_{2 i} \in[\dot{\phi}, \dot{\theta}, \dot{\psi}]^{T}$.

3.2. Design of Integral Sliding Mode Control. The design of a sliding mode controller is typically composed of two steps. The first step features the construction of a sliding surface, on which the desired system performance can be maintained. The second step is concerned with the selection of the appropriate control law to force the sliding variable to reach the designed sliding surface, and hereafter, keep the sliding motion within the close neighborhood of the designed sliding surface.

First, denote $x_{2 i-1}^{d}$ and $x_{2 i}^{d}$ as the desired trajectories. Then, the tracking errors can be defined as $\tilde{x}_{i 1}=x_{2 i-1}-$ $x_{2 i-1}^{d}$ and $\tilde{x}_{i 2}=x_{2 i}-x_{2 i}^{d}$. In this sense, the integral sliding surface for the system is defined by the following set as [28]

$$
\left\{\begin{array}{l}
\sigma_{i}=c_{i} \tilde{x}_{i 1}+\tilde{x}_{i 2}, \\
\dot{z}_{i}=-c_{i} \tilde{x}_{i 2}+k_{i 2} \tilde{x}_{i 2}+k_{i 1} \tilde{x}_{i 1}, \\
z_{i}(0)=-c_{i} \tilde{x}_{i 1}\left(t_{0}\right)-\tilde{x}_{i 2}\left(t_{0}\right),
\end{array}\right.
$$

where $\sigma_{i}$ is the nonlinear combination of the tracking errors, $z_{i}$ includes the integral term, $t_{0}$ is the initial time instant, and $k_{i 1}, k_{i 2}$, and $c_{i}$ are the design parameters.

In this case, by combining the equations in (6), the integral sliding surface can be described as

$s_{i}=\sigma_{i}+z_{i}=\tilde{x}_{i 2}+k_{i 2} \tilde{x}_{i 1}+k_{i 1} \int_{t_{0}}^{t} \tilde{x}_{i 1}(\tau) d \tau-k_{i 2} \tilde{x}_{i 1}\left(t_{0}\right)-\tilde{x}_{i 2}\left(t_{0}\right)$.

The next step is to design an appropriate control law to keep the sliding variable within the close neighborhood of the defined sliding surface. The sliding mode control law is usually constructed with two control portions, i.e., the continuous control portion and the discontinuous control portion. The continuous control portion is derived by equalizing $\dot{s}_{i}=0$, and the disturbance $d_{i}$ is not considered at this time. In this case, the continuous control portion can be designed as

$$
v_{i 1}=h_{i}^{-1}\left(\ddot{x}_{2 i-1}^{d}-k_{i 2} \tilde{x}_{i 2}-k_{i 1} \tilde{x}_{i 1}-f_{i}(x)\right) \text {. }
$$

Then, in order to compensate the disturbance $d_{i}$, the discontinuous control portion is designed and synthesized to ensure the desired sliding motion as

$$
v_{i 2}=-k_{i 3} \operatorname{sign}\left(s_{i}\right) \text {, }
$$

where $k_{i 3}$ is a positive gain that makes the sliding surface attractive to the sliding variable.

However, for achieving the disturbance rejection capability, a large value of $k_{i 3}$ is required. This can increase the discontinuity of sliding mode control and may lead to unexpected control chattering. A common approach for smoothing the discontinuity is to add a thin boundary layer neighboring the defined sliding surface. The boundary layer can be formulated as [29]

$$
\bar{B}=\left\{\tilde{x}_{i 1}, \tilde{x}_{i 2},\left|s_{i}\right| \leq \Phi_{i}\right\}
$$

where $\Phi_{i}$ represents the thickness of the boundary layer.

By employing the defined boundary layer, a saturation function for replacing the sign function in Equation (9) can be defined as

$$
\operatorname{sat}\left(s_{i}\right)= \begin{cases}1 & \text { if } s_{i}>\Phi_{i} \\ -1 & \text { if } s_{i}<-\Phi_{i} \\ s_{i} / \Phi_{i} & \text { if }\left|s_{i}\right| \leq \Phi_{i}\end{cases}
$$

Accordingly, by combining both the continuous and discontinuous control portions, the developed control law can be formulated as

$$
v_{i}=h_{i}^{-1}\left(\ddot{x}_{2 i-1}^{d}-k_{i 2} \tilde{x}_{i 2}-k_{i 1} \tilde{x}_{i 1}-f_{i}(x)\right)-k_{i 3} \operatorname{sat}\left(s_{i}\right) .
$$




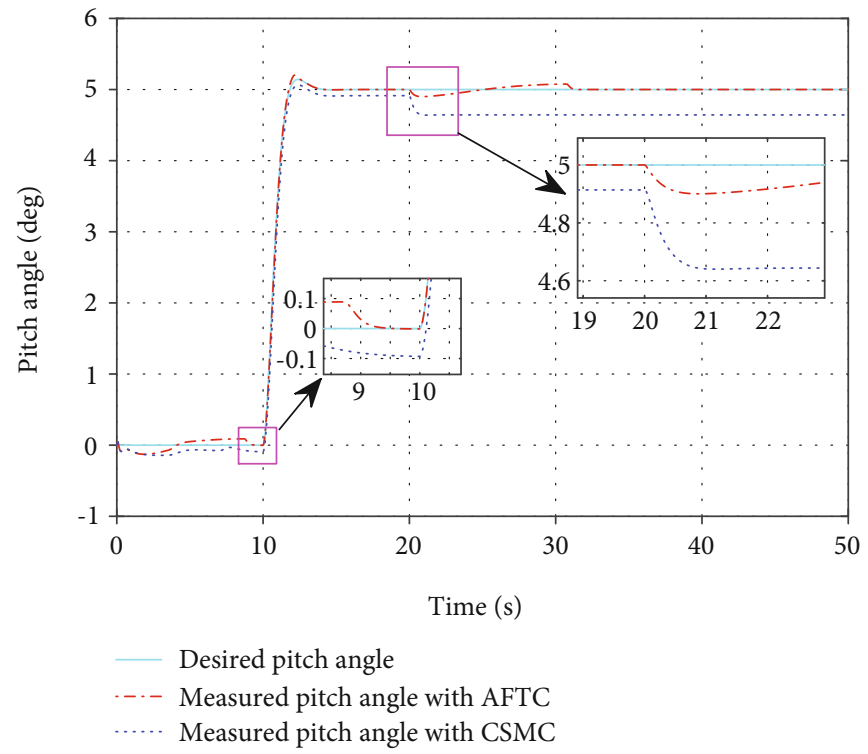

FIGURE 3: Pitch motion tracking performance of the UAV in the presence of elevator fault in scenario 1.

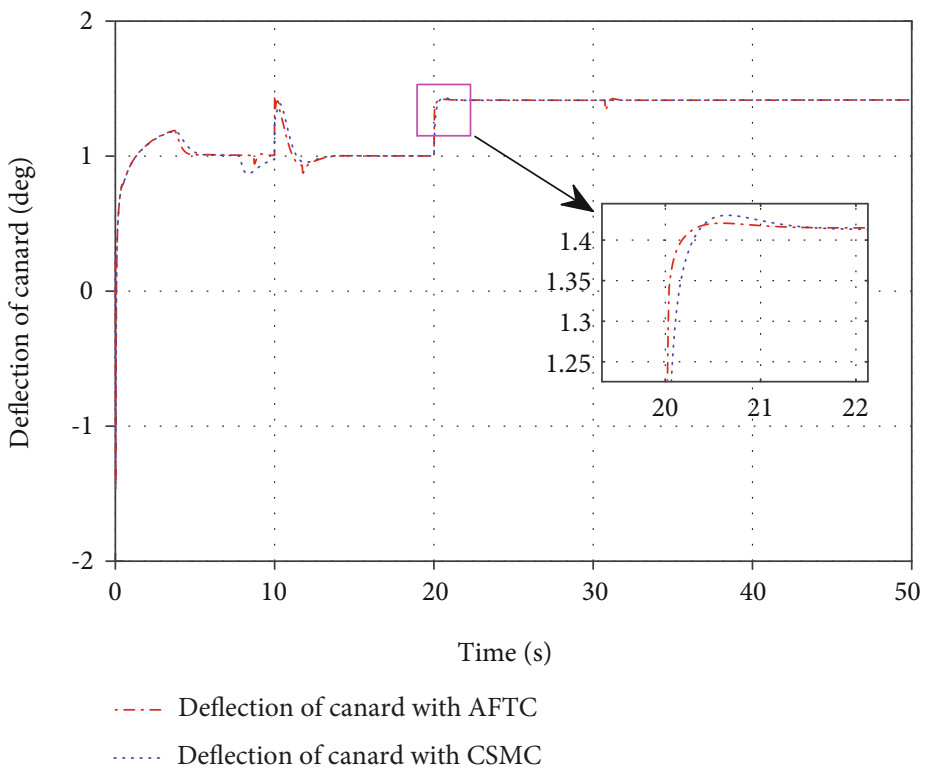

Figure 4: Deflection of canard control surface in the presence of elevator fault in scenario 1.

3.3. Adaptive Sliding Mode Control. Since the studied hybrid CRW UAV is an overactuated system, when actuator faults occur, one needs to obtain the fault information and accordingly redistribute the control signals over the available actuators. Considering the implementation of the control scheme in a real system, the control reallocation needs to be triggered instantly when actuator faults occur. Given the considered system in Equation (5), the actual control input $u$ is computed by employing a quadratic optimization approach, such that the actual virtual control signal generated from the control allocation module can meet the requirement of the desired virtual control signal produced by the high-level sliding mode control.
The quadratic programming approach based on minimizing control input can be described as

$$
\begin{gathered}
J=\arg \min _{u} u^{T} W u \\
\text { s.t. } \quad v_{i}=B_{u i} u
\end{gathered}
$$

It has an explicit solution as follows:

$$
u=W B_{u i}^{T}\left(B_{u i} W B_{u i}^{T}\right)^{-1} v_{i}
$$

where $W=W^{T}=\operatorname{diag}\left(\left[w_{1}, w_{2}, \cdots, w_{m}\right]\right)$ is a symmetric 


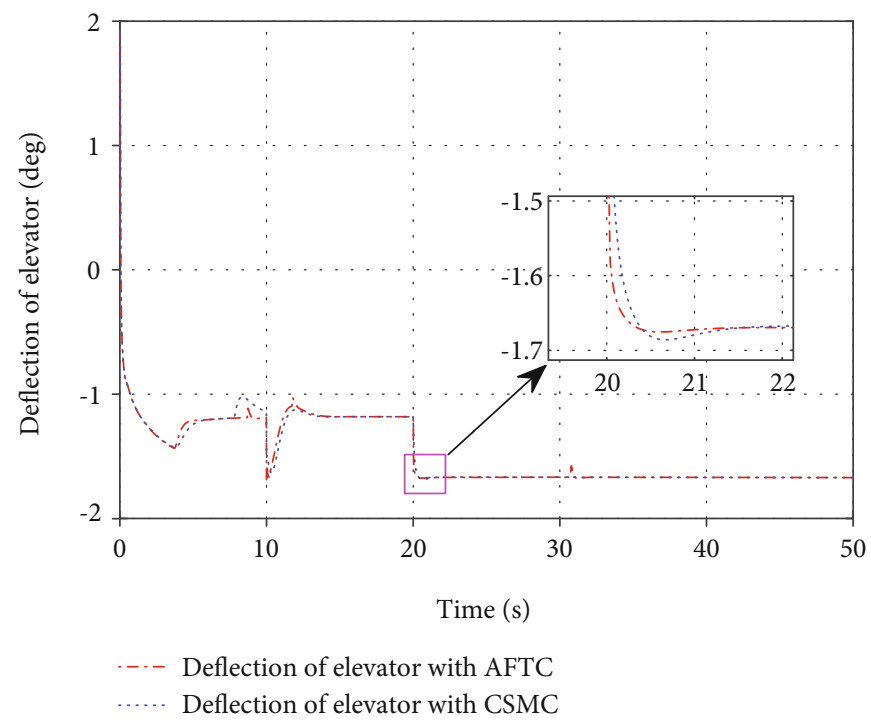

FIgURE 5: Deflection of elevator in the presence of elevator fault in scenario 1.

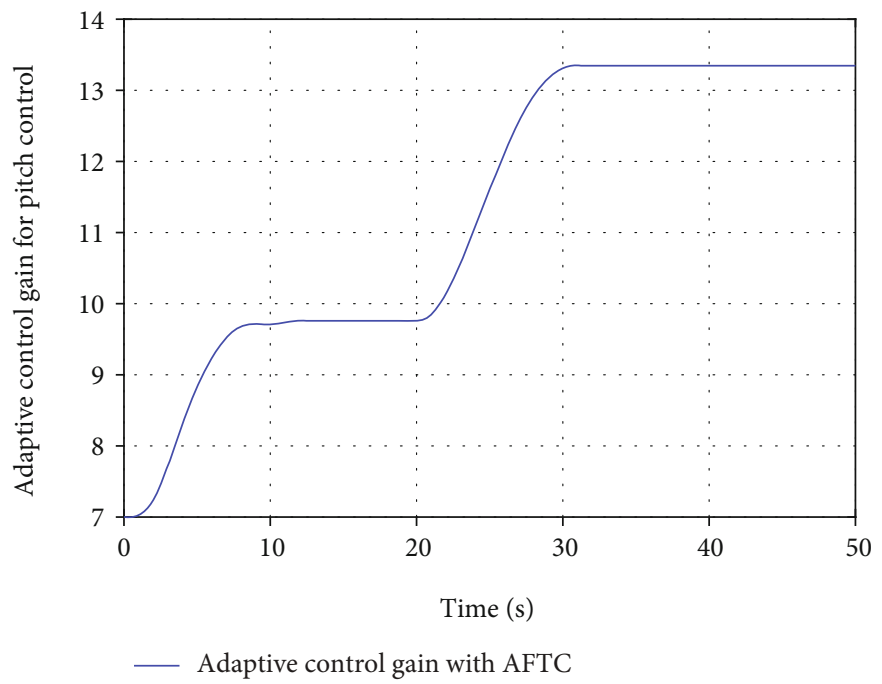

FIGURE 6: Change of adaptive control parameter in the presence of elevator fault in scenario 1.

positive definite weighting matrix that is related to the remaining control effectiveness of the actuators.

The commonly used approach for achieving faulttolerant control of an overactuated system is to change the weighting matrix $W$, which requires fault information from the so-called fault detection and diagnosis (FDD) module. However, in practical applications, there may exist large time delay to obtain the required fault information. In addition, there may also exist fault estimation errors, which will affect the corresponding control performance as well. In this case, an adaptive control strategy is proposed to accommodate actuator faults for the overactuated system without requiring any fault information.

In the case of actuator faults, the diagonal matrix $W$ will not be an identity matrix. Since the actual fault information cannot be obtained, if the matrix $W$ is still chosen as an identity matrix for computing the actual control input $u$, there will exist virtual control errors. In this condition, the following equation can be obtained:

$$
v_{i}=B_{u i} I u-B_{u i}\left(I-L_{f}\right) u
$$

where $I$ is an identity matrix.

Let $v_{i e}=-B_{u i}\left(I-L_{f}\right) u$, the system dynamics in Equation (5) can be rewritten as

$$
\dot{x}_{2 i}=f_{i}(x)+h_{i}\left(v_{i d}+v_{i e}\right)+d_{i},
$$

where $v_{i d}=B_{u i} I u$ represents the desired virtual control signal from the high-level controller. 
As can be observed from Equation (16), the parameter $h_{i}$ needs to be adaptively adjusted to eliminate the virtual control signal error $v_{i e}$ in order to maintain the closedloop system performance. With this consideration, let $h_{i}$ $v_{i e}$ be equal to $\tilde{h}_{i} v_{i d}$, thus, Equation (16) can be reconstructed as

$$
\dot{x}_{2 i}=f_{i}(x)+\left(h_{i}+\tilde{h}_{i}\right) v_{i d}+d_{i}=f_{i}(x)+\widehat{h}_{i} v_{i d}+d_{i}
$$

After actuator faults occur, the actual generated virtual control signal from the control allocation module will not be equal to the desired virtual control signal generated from the high-level controller. Under this condition, by adaptively changing the parameter $\widehat{h}_{i}$, more virtual control signals will be generated from the high-level controller, which can help to compensate the adverse effect caused by actuator faults and maintain the original tracking performance of the closed-loop system. In addition, to fully use both continuous and discontinuous control portions of sliding mode control, the estimated parameter $\widehat{h}_{i}$ is added to the discontinuous control portion and makes adaptive change of both continuous and discontinuous control portions of sliding mode control in the presence of actuator faults. Moreover, with consideration of model uncertainties and actuator faults, both estimations $\widehat{f}_{i}$ and $\widehat{h}_{i}$ should be employed for deriving the corresponding high-level control law. In this case, the previously developed control law in Equation (12) can be reconstructed as

$$
\begin{aligned}
v_{i} & =\widehat{h}_{i}^{-1}\left(\ddot{x}_{2 i-1}^{d}-k_{i 2} \tilde{x}_{i 2}-k_{i 1} \tilde{x}_{i 1}-\widehat{f}_{i}(x)\right)-\widehat{h}_{i}^{-1} k_{i 3} \operatorname{sat}\left(s_{i}\right), \\
u & =B_{u i}^{T}\left(B_{u i} B_{u i}^{T}\right)^{-1} v_{i} .
\end{aligned}
$$

Then, by denoting $\widehat{\chi}_{i}=\widehat{h}_{i}^{-1} \widehat{f}_{i}(x)$ and $\widehat{\Upsilon}_{i}=\widehat{h}_{i}^{-1}$, the developed control law in Equation (18) can be rearranged as

$$
u=B_{u i}^{+} \widehat{\gamma}_{i}\left(\ddot{x}_{2 i-1}^{d}-k_{i 2} \tilde{x}_{i 2}-k_{i 1} \tilde{x}_{i 1}-k_{i 3} \operatorname{sat}\left(s_{i}\right)\right)-B_{u i}^{+} \widehat{\chi}_{i},
$$

where $B_{u i}^{+}=B_{u i}^{T}\left(B_{u i} B_{u i}^{T}\right)^{-1}$.

Accordingly, in order to compensate both actuator faults and model uncertainties, the estimated parameters $\widehat{\chi}_{i}$ and $\widehat{\gamma}_{i}$ need to be updated online. For this purpose, the corresponding online adaptive schemes for estimating the uncertain parameters are designed as

$$
\begin{gathered}
\dot{\hat{\chi}}_{i}=\Delta s_{i}, \\
\dot{\hat{\Upsilon}}_{i}=\left(k_{i 1} \tilde{x}_{i 1}+k_{i 2} \tilde{x}_{i 2}-\dot{x}_{2 i}^{d}+k_{i 3} \operatorname{sat}\left(s_{i}\right)\right) \Delta s_{i},
\end{gathered}
$$

where $\Delta s_{i}=s_{i}-\Phi_{i} \operatorname{sat}\left(s_{i}\right)$ represents the distance between the sliding variable and the defined boundary layer. Only when the sliding variable is outside the defined boundary layer, i.e., the system tracking performance is unsatisfactory, the adaptive schemes can be triggered. By defining such a variable, the overestimation of the uncertain parameters can be avoided, which further contributes to alleviating system control chattering.

Theorem 1. Consider an integral chain nonlinear affine system with actuator faults, model uncertainties, and bounded disturbances in Equation (4). Given the defined integral sliding surface in Equation (7), by designing the feedback control law in Equation (19), and the corresponding online adaptive schemes in Equation (20), the sliding variable will be driven to reach the defined sliding surface and thereafter kept inside the boundary layer even in the presence of actuator faults, model uncertainties, and disturbances by choosing the discontinuous control gain as $k_{i 3} \geq \eta_{i}+D_{i}$.

Proof of Theorem 1. Consider the following Lyapunov candidate function as

$$
V=\sum_{i=1}^{3} \frac{1}{2}\left[\Delta s_{i}^{2}+Y_{i}^{-1}\left(\chi \wedge_{i}-\chi_{i}\right)^{2}+Y_{i}^{-1}\left(Y \wedge_{i}-Y_{i}\right)^{2}\right]
$$

The derivative of the selected Lyapunov candidate function can be calculated as

$$
\begin{aligned}
& \dot{V}=\sum_{i=1}^{3}\left[\Delta s_{i} \Delta \dot{s}_{i}+Y_{i}^{-1}\left(\widehat{\chi}_{i}-\chi_{i}\right) \dot{\hat{\chi}}+Y_{i}^{-1}\left(\hat{\Upsilon}_{i}-Y_{i}\right) \dot{\hat{\Upsilon}}\right] \\
& =\sum_{i=1}^{3}\left[\Delta s_{i}\left(\dot{\tilde{x}}_{i 2}+k_{i 2} \dot{\tilde{x}}_{i 1}+k_{i 1} \tilde{x}_{i 1}\right)+Y_{i}^{-1}\left(\widehat{\chi}_{i}-\chi_{i}\right) \dot{\hat{\chi}}\right. \\
& \left.+\Upsilon_{i}^{-1}\left(\widehat{\Upsilon}_{i}-\gamma_{i}\right) \dot{\hat{\Upsilon}}\right]=\sum_{i=1}^{3}\left[\Delta s_{i}\left(\dot{x}_{2 i}-\dot{x}_{2 i}^{d}+k_{i 2} \dot{\tilde{x}}_{i 1}+k_{i 1} \tilde{x}_{i 1}\right)\right. \\
& \left.+Y_{i}^{-1}\left(\widehat{\chi}_{i}-\chi_{i}\right) \dot{\hat{\chi}}+Y_{i}^{-1}\left(\widehat{\Upsilon}_{i}-Y_{i}\right) \dot{\hat{\Upsilon}}\right] \\
& =\sum_{i=1}^{3}\left[\Delta s_{i}\left(f_{i}(x)+h_{i} B_{u} u+d_{i}-\dot{x}_{2 i}^{d}+k_{i 2} \dot{\tilde{x}}_{i 1}+k_{i 1} \tilde{x}_{i 1}\right)\right. \\
& \left.+Y_{i}^{-1}\left(\widehat{\chi}_{i}-\chi_{i}\right) \dot{\hat{\chi}}+Y_{i}^{-1}\left(\widehat{\Upsilon}_{i}-Y_{i}\right) \dot{\hat{\Upsilon}}\right] \\
& =\sum_{i=1}^{3}\left[\Delta s _ { i } \left(Y_{i}^{-1} \chi_{i}+Y_{i}^{-1} B_{u} B_{u i}^{+} \widehat{\Upsilon}_{i}\left(\ddot{x}_{2 i-1}^{d}-k_{i 2} \tilde{x}_{i 2}-k_{i 1} \tilde{x}_{i 1}-k_{i 3} \operatorname{sat}\left(s_{i}\right)\right)\right.\right. \\
& \left.-Y_{i}^{-1} B_{u} B_{u i}^{+} \widehat{\chi}_{i}+d_{i}-\dot{x}_{2 i}^{d}+k_{i 2} \dot{\tilde{x}}_{i 1}+k_{i 1} \tilde{x}_{i 1}\right) \\
& \left.+\gamma_{i}^{-1}\left(\widehat{\chi}_{i}-\chi_{i}\right) \dot{\hat{\chi}}+Y_{i}^{-1}\left(\widehat{\Upsilon}_{i}-r_{i}\right) \dot{\hat{\gamma}}\right] \\
& =\sum_{i=1}^{3}\left[\Delta s _ { i } \left[\Upsilon_{i}^{-1} \chi_{i}-\Upsilon_{i}^{-1} \widehat{\chi}_{i}+\Upsilon_{i}^{-1} \widehat{\Upsilon}_{i}\left(\dot{x}_{2 i}^{d}-k_{i 2} \tilde{x}_{i 2}-k_{i 1} \tilde{x}_{i 1}\right)\right.\right. \\
& \left.-\Upsilon_{i}^{-1} \widehat{\Upsilon}_{i} k_{i 3} \operatorname{sat}\left(s_{i}\right)+d_{i}-\left(\dot{x}_{2 i}^{d}-k_{i 2} \tilde{x}_{i 2}-k_{i 1} \tilde{x}_{i 1}\right)\right] \\
& \left.+\gamma_{i}^{-1}\left(\widehat{\chi}_{i}-\chi_{i}\right) \dot{\hat{\chi}}+Y_{i}^{-1}\left(\widehat{\Upsilon}_{i}-\gamma_{i}\right) \dot{\hat{\gamma}}\right] \\
& =\sum_{i=1}^{3}\left[\left(Y_{i}^{-1} \widehat{\Upsilon}_{i}-1\right)\left[\dot{\hat{\Upsilon}}+\left(\dot{x}_{2 i}^{d}-k_{i 2} \tilde{x}_{i 2}-k_{i 1} \tilde{x}_{i 1}-k_{i 3} \operatorname{sat}\left(s_{i}\right)\right) \Delta s_{i}\right]\right. \\
& \left.+Y_{i}^{-1}\left(\chi_{i}-\widehat{\chi}_{i}\right)\left(\Delta s_{i}-\dot{\hat{\chi}}\right)+\left(d_{i}-k_{i 3} \operatorname{sat}\left(s_{i}\right)\right) \Delta s_{i}\right] .
\end{aligned}
$$




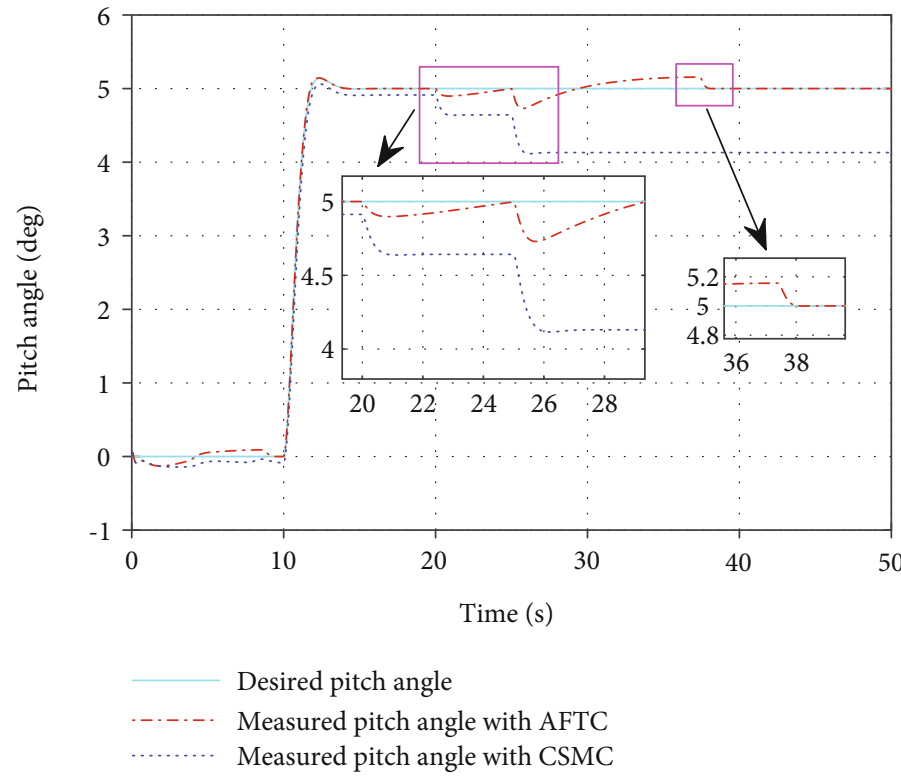

FIGURE 7: Pitch motion tracking performance of the UAV in the presence of concurrent elevator and canard control surface faults in scenario 2 .

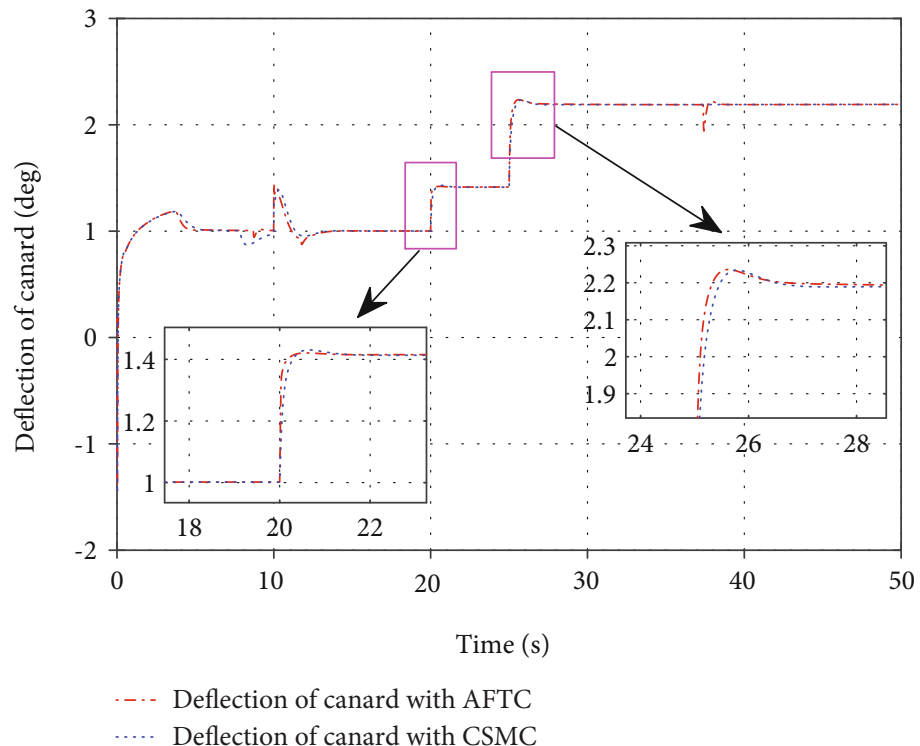

Figure 8: Deflection of canard control surface in the presence of concurrent elevator and canard control surface faults in scenario 2.

Then, by substituting Equation (20) into Equation (22), it leads to

$$
\begin{aligned}
\dot{V} & =\sum_{i=1}^{3}\left[d_{i} \Delta s_{i}-k_{i 3} \operatorname{sat}\left(s_{i}\right) \Delta s_{i}\right] \\
& \leq \sum_{i=1}^{3}\left[D_{i} \Delta s_{i}-\left(\eta_{i}+D_{i}\right) \operatorname{sat}\left(s_{i}\right) \Delta s_{i}\right] \\
& \leq \sum_{i=1}^{3}-\eta_{i}\left|\Delta s_{i}\right| .
\end{aligned}
$$

Therefore, with the proposed control law and adaptive schemes, the system stability can be maintained in the presence of actuator faults, model uncertainties, and bounded disturbances.

\section{Simulation Results}

In order to demonstrate the performance of the proposed adaptive fault-tolerant control (AFTC) scheme, simulations based on the studied over-actuated hybrid CRW UAV in fixed-wing flight mode under different uncertain and faulty scenarios are carried out. For the purpose of comparison, a conventional sliding mode control (CSMC) without adaptation is also demonstrated as the high-level controller 


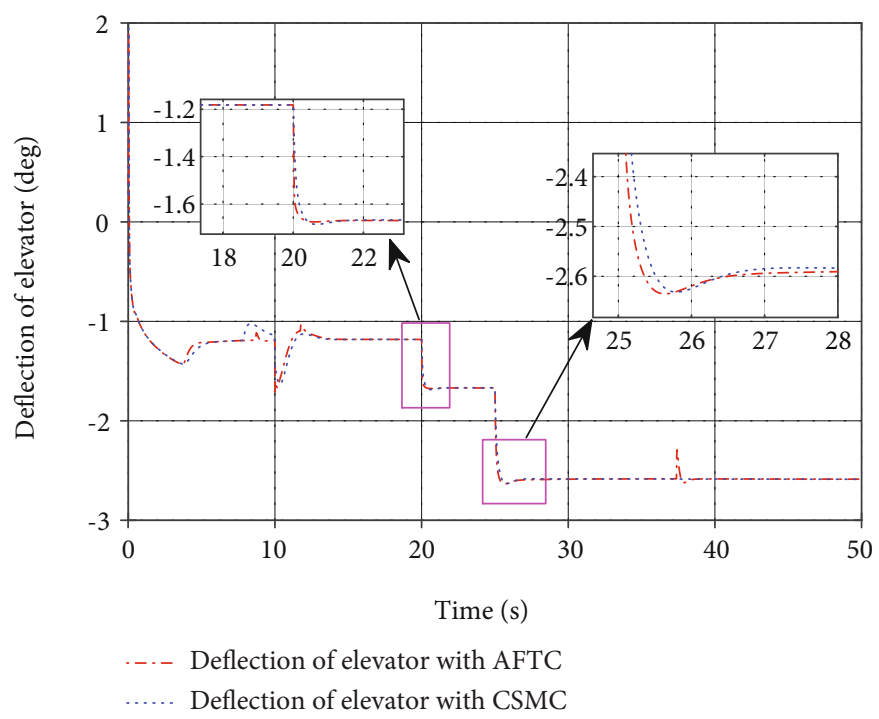

Figure 9: Deflection of elevator in the presence of concurrent elevator and canard control surface faults in scenario 2.

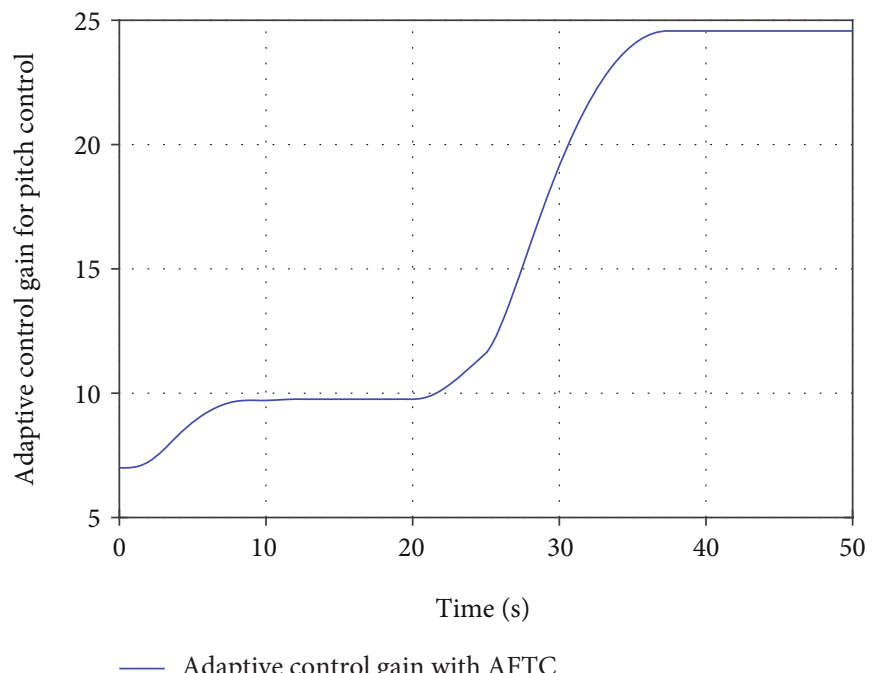

FIGURE 10: Change of adaptive control parameter in the presence of concurrent elevator and canard control surface faults in scenario 2.

combined with the low-level control allocation module in the following simulation scenarios. The control parameters are chosen as $k_{11}=10, k_{21}=10, k_{31}=8, k_{12}=25, k_{22}=25$, $k_{32}=16, k_{13}=1, k_{23}=1, k_{33}=1, \eta_{1}=\eta_{2}=\eta_{3}=0.1, \Phi_{1}=\Phi_{2}$ $=\Phi_{3}=0.05$.

The UAV is controlled to track a set of predesigned pitch commands which are generated from the following prefilter:

$$
\ddot{\theta}_{d}+3 \dot{\theta}_{d}+4 \theta_{d}=4 \theta^{\star}
$$

where $\theta^{\star}$ changes from 0 to 5 degree at $10 \mathrm{~s}$.

In addition to the uncertain portions of the resultant torques acting on the studied UAV, the uncertainties corresponding to the roll, pitch, and yaw moment of inertia are set as $10 \%$ of their measured values. Focusing on the fault pattern of the actuators, two faulty scenarios are investigated in this section. In the first scenario, a 50\% loss of control effectiveness fault is injected to elevator at $20 \mathrm{~s}$. In the second scenario, in addition to the fault occurred in elevator, a $60 \%$ loss of control effectiveness fault is injected to canard control surface at $25 \mathrm{~s}$.

In scenario 1 , a $50 \%$ loss of control effectiveness fault is considered occurring in elevator. The tracking performance of pitch motion of the UAV is shown in Figure 3. It can be observed that when there is no fault (before $20 \mathrm{~s}$ ), the proposed AFTC can track the desired pitch angle with no tracking error. On the contrary, the compared CSMC tracks the desired pitch angle with a small tracking error. This is because due to the existence of unknown torques, the proposed AFTC can adaptively change the control parameter to strive for accurate tracking performance. When actuator fault occurs at $20 \mathrm{~s}$, by adaptively changing the control parameter, the proposed AFTC can still maintain the original tracking performance for pitch motion. Nevertheless, 
the compared CSMC fails to maintain the original tracking performance. The control inputs of canard control surface and elevator are illustrated in Figures 4 and 5. When elevator experiences fault, the control inputs of both elevator and canard control surface will be increased correspondingly to eliminate the adverse impact of fault. In addition, in order for compensating the fault, the use of canard control surface and elevator is almost the same for the proposed AFTC and the compared CSMC, which indicates that the proposed control scheme does not use more control effort for compensating the fault. Figure 6 shows the change of adaptive control parameters of the proposed AFTC scheme.

In scenario 2, in order to further investigate the performance of the proposed control strategy, in addition to the $50 \%$ partial loss fault in elevator occurring at $20 \mathrm{~s}$, a $60 \%$ loss of control effectiveness fault is injected to canard control surface at $25 \mathrm{~s}$. The tracking performance of pitch motion is shown in Figure 7. For the pitch motion control, after faults occur, the proposed AFTC can make a quick compensation to maintain the desired tracking performance, where the compared CSMC fails to maintain the desired tracking performance and the tracking error increases with the fault becoming larger. The control inputs of elevator and canard control surface are shown in Figures 8 and 9. Compared to scenario 1 , due to the additional fault in canard control surface, the use of elevator and canard control surface is also increased. This can also be observed from Figure 10 where in order to account for larger fault, the value of the adaptive control parameter is increased as well.

\section{Conclusions}

In this paper, an adaptive fault-tolerant control scheme is proposed for an overactuated hybrid CRW UAV to compensate concurrent actuator faults and model uncertainties without the requirement of fault and uncertainty information. The proposed control scheme is divided into two separate control modules: the high-level adaptive sliding mode control module and the low-level control allocation module. The low-level control allocation module is used to distribute the virtual control signals that are generated by the highlevel control module among the redundant available actuators. The high-level control module is constructed by an adaptive sliding mode controller, which is employed to maintain the overall system tracking performance in both faulty and uncertain conditions. In the case of actuator faults and model uncertainties, the adaptive scheme will be triggered to generate more virtual control signals. With the help of the synthesized adaptive schemes, the control parameters can be changed adaptively to compensate the virtual control errors. The comparative simulation results show the effectiveness and advantages of the proposed strategy in the presence of both single and concurrent actuator faults.

\section{Data Availability}

The data used to support the findings of this study are included within this article.

\section{Conflicts of Interest}

The authors declare that there is no conflict of interest regarding the publication of this paper.

\section{Acknowledgments}

This research was partially supported by the National Natural Science Foundation of China under Grant no. 62003266 and the Fundamental Research Funds for the Central Universities. The presentation of the article is partially based on the thesis authored by Ban Wang.

\section{References}

[1] F. A. Goodarzi, D. Lee, and T. Lee, "Geometric control of a quadrotor UAV transporting a payload connected via flexible cable," International Journal of Control, Automation and Systems, vol. 13, no. 6, pp. 1486-1498, 2015.

[2] J. Chen, H. Liu, J. Zheng et al., "Damage degree evaluation of earthquake area using UAV aerial image," International Journal of Aerospace Engineering, vol. 2016, Article ID 2052603, 10 pages, 2016.

[3] Y. Liu, Z. Liu, J. Shi, G. Wu, and C. Chen, "Optimization of base location and patrol routes for unmanned aerial vehicles in border intelligence, surveillance, and reconnaissance," Journal of Advanced Transportation, vol. 2019, Article ID 9063232, 13 pages, 2019.

[4] A. S. Saeed, A. B. Younes, C. Cai, and G. W. Cai, "A survey of hybrid unmanned aerial vehicles," Progress in Aerospace Sciences, vol. 98, pp. 91-105, 2018.

[5] Y. Zhou, G. Huang, and C. Xia, "Analysis of fixed-wing VTOL aircraft with gas-driven fan propulsion system," Aerospace Science and Technology, vol. 104, article 105984, 2020.

[6] A. Kamal and A. Ramirez-Serrano, "Conceptual design of a highly-maneuverable transitional VTOL UAV with new maneuver and control capabilities," in AIAA Scitech 2020 Forum, Orlando, FL, 2020.

[7] U. Ozdemir, Y. O. Aktas, A. Vuruskan et al., "Design of a commercial hybrid VTOL UAV system," Journal of Intelligent \& Robotic Systems, vol. 74, no. 1-2, pp. 371-393, 2014.

[8] A. Oosedo, S. Abiko, A. Konno, T. Koizumi, T. Furui, and M. Uchiyama, "Development of a quad rotor tail-sitter VTOL UAV without control surfaces and experimental verification," in Proceedings of IEEE International Conference on Robotics and Automation, pp. 317-322, Karlsruhe, Germany, 2013.

[9] B. Yuksek, A. Vuruskan, U. Ozdemir, M. A. Yukselen, and G. Inalhan, "Transition flight modeling of a fixed-wing VTOL UAV," Journal of Intelligent \& Robotic Systems, vol. 84, no. 1-4, pp. 83-105, 2016.

[10] D. Rohr, M. Studiger, T. Stastny, N. Lawrance, and R. Siegwart, "Nonlinear model predictive velocity control of a VTOL tiltwing UAV," IEEE Robotics and Automation Letters, vol. 6, no. 3, pp. 5776-5783, 2021.

[11] H. Gao, A. He, Z. Gao, Y. Na, and Y. Deng, "Flight dynamics characteristics of canard rotor/wing aircraft in helicopter flight mode," Chinese Journal of Aeronautics, vol. 32, no. 7, pp. 1577-1587, 2019.

[12] Z. Li, L. Zhang, H. Liu, Z. Zuo, and C. Liu, "Nonlinear robust control of tail-sitter aircrafts in flight mode transitions," Aerospace Science and Technology, vol. 81, pp. 348-361, 2018. 
[13] N. Liu, Z. Cai, Y. Wang, and J. Zhao, "Fast level-flight to hover mode transition and altitude control in tiltrotor's landing operation," Chinese Journal of Aeronautics, vol. 34, no. 1, pp. 181-193, 2021.

[14] Z. Liu, J. Guo, M. Li, S. Tang, and X. Wang, "VTOL UAV transition maneuver using incremental nonlinear dynamic inversion," International Journal of Aerospace Engineering, vol. 2018, Article ID 6315856, 19 pages, 2018.

[15] B. Xian, G. Jianchuan, Z. Yao, and Z. Bo, "Sliding mode tracking control for miniature unmanned helicopters," Chinese Journal of Aeronautics, vol. 28, no. 1, pp. 277-284, 2015.

[16] K. Oner, E. Cetinsoy, E. Sirimoglu, C. Hancer, T. Ayken, and M. Unel, "LQR and SMC stabilization of a new unmanned aerial vehicle," World Academy of Science, Engineering and Technology, vol. 3, no. 10, pp. 367-372, 2009.

[17] B. Wang, X. Yu, L. Mu, and Y. M. Zhang, "Disturbance observer-based adaptive fault-tolerant control for a quadrotor helicopter subject to parametric uncertainties and external disturbances," Mechanical Systems and Signal Processing, vol. 120, pp. 727-743, 2019.

[18] C. Tan, G. Xu, L. Dong, H. Zhao, J. Li, and S. Zhang, "Neural network-based finite-time fault-tolerant control for spacecraft without unwinding," International Journal of Aerospace Engineering, vol. 2021, Article ID 9269438, 10 pages, 2021.

[19] M. Qian, Y. Shi, Z. Gao, and X. Zhang, "Integrated fault tolerant tracking control for rigid spacecraft using fractional order sliding mode technique," Journal of the Franklin Institute, vol. 357, no. 15, pp. 10557-10583, 2020.

[20] F. Caliskan, Y. M. Zhang, N. E. Wu, and J. Y. Shin, “Actuator fault diagnosis in a Boeing 747 model via adaptive modified two-stage Kalman filter," International Journal of Aerospace Engineering, vol. 2014, Article ID 472395, 10 pages, 2014.

[21] Y. Yu and Y. Q. Dong, "Global fault-tolerant control of underactuated aerial vehicles with redundant actuators," International Journal of Aerospace Engineering, vol. 2019, Article ID 9754981, 10 pages, 2019.

[22] S. Fuhrer, S. Verling, T. Stastny, and R. Siegwart, "Fault-tolerant flight control of a VTOL tailsitter UAV," in Proceedings of International Conference on Robotics and Automation, Montreal, QC, Canada, 2019.

[23] S. Park, J. Bae, Y. Kim, and S. Kim, "Fault tolerant flight control system for the tilt-rotor UAV," Journal of the Franklin Institute, vol. 350, no. 9, pp. 2535-2559, 2013.

[24] M. Qian and X. G. Yan, "Integrated fault-tolerant control approach for linear time-delay systems using a dynamic event-triggered mechanism," International Journal of Systems Science, vol. 51, no. 16, pp. 3471-3490, 2020.

[25] Z. Yu, Z. Liu, Y. M. Zhang, Y. Qu, and C.-Y. Su, "Distributed finite-time fault-tolerant containment control for multiple unmanned aerial vehicles," IEEE Transactions on Neural Networks and Learning Systems, vol. 31, no. 6, pp. 2077-2091, 2020.

[26] Z. Yu, Y. M. Zhang, B. Jiang, J. Fu, Y. Jin, and T. Chai, "Composite adaptive disturbance observer-based decentralized fractional-order fault-tolerant control of networked UAVs," IEEE Transactions on Systems, Man, and Cybernetics: Systems, pp. 1-15, 2021.

[27] W. Gai, J. Liu, J. Zhang, and Y. Li, "A new closed-loop control allocation method with application to direct force control," International Journal of Control, Automation and Systems, vol. 16, no. 3, pp. 1355-1366, 2018.
[28] V. Utkin and J. Shi, "Integral sliding mode in systems operating under uncertainty conditions," in Proceedings of the 35th Conference on Decision and Control, pp. 4591-4596, Kobe, Japan, 1996.

[29] B. Wang, Fault Diagnosis and Fault-Tolerant Control of Unmanned Aerial Vehicles, [Ph.D. thesis], Concordia University, 2018. 